\title{
Power Spectral Density Analysis of Electrocorticogram Recordings during Cerebral Hypothermia in Neonatal Seizures
}

\author{
Mark H. Myers ${ }^{a}$ Elliott Jolly ${ }^{b}$ Yaqin $\mathrm{Li}^{\mathrm{c}}$ Amy de Jongh Curry ${ }^{\mathrm{b}}$ \\ Helena Parfenovad

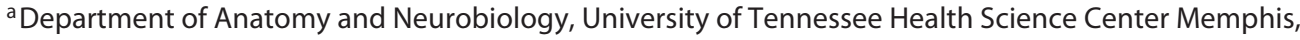 \\ ${ }^{b}$ Department of Biomedical Engineering, University of Memphis, ' ${ }^{\mathrm{C}}$ Department of Ophthalmology, \\ University of Tennessee Health Science Center, and ${ }^{\mathrm{d}}$ Department of Physiology, University of \\ Tennessee Health Science Center, Memphis, TN, USA
}

\section{Keywords}

Power spectral density · Electrocorticogram · Seizure activity · Cerebral hypothermia · Beta-gamma activity

\begin{abstract}
Background: Neonatal seizures (NS) are the most common form of neurological dysfunction observed in newborns. Purpose: The purpose of this study in newborn piglets was to determine the effect of cerebral hypothermia $(\mathrm{CH})$ on neural activity during pharmacologically induced NS. We hypothesized that the neuroprotective effects of $\mathrm{CH}$ would preserve higher frequencies observed in electrocorticogram (ECoG) recordings. Methods: Power spectral density was employed to determine the levels of brain activity in ECoGs to quantitatively assess the power of each frequency observed in neurological brain states of delta, theta, alpha, and beta-gamma frequencies. Result: The most significant reduction of power occurs in the lower frequency band of delta-theta-alpha of $\mathrm{CH}$ cohorts, while $t$ score probabilities imply that high-frequency brain activity in the beta-gamma range is preserved in the $\mathrm{CH}$ population. Conclusion: While the overall power density decreases over time in both groups, the decrease is to a lesser degree in the $\mathrm{CH}$ population.

(c) 2017 S. Karger AG, Basel
\end{abstract}

\section{Introduction}

Neonatal seizures (NS) are the most common form of neurological dysfunction observed in newborns. Generally, these events can serve as precursors for other forms of brain disorders that develop later in life [1]. Seizures occur when a large group of neurons fire in a synchronous and excessive manner. This deleterious activity can occur for many reasons, for example due to the imbalance in excitatory and/or inhibitory neurotransmitters (e.g., gamma amino butyric acid [GABA]). Hypoxic-ischemic encephalopathy (HIE) is a prominent cause of seizures in infants. As oxygen is necessary for the production of adenosine triphosphate (ATP), HIE results in the collapse of ATP-dependent resting membrane potentials [1]. In a study by Gunn et al. [2], damage from experimental HIE has been proven to be effectively reduced by cerebral hypothermia $(\mathrm{CH})$ if started shortly after reperfusion. The purpose of this study was to determine if there is any effect of $\mathrm{CH}$ on pharmacologically induced NS. It was hypothesized that the neuroprotective effects of $\mathrm{CH}$ would preserve 13-100 Hertz $(\mathrm{Hz})$ neural activity associated with cognitive processing [3], observed in electrocorticogram (ECoG) recordings. Hypothermia was initiated $20 \mathrm{~min}$ prior to the initiation of seizures to ensure

\section{KARGER}

(c) 2017 S. Karger AG, Basel 
hypothermia was reached. Piglets 3-7 days old were used to experimentally model the neonatal brain due to their fully developed cerebral cortices, high level of physiological similarities and comparable brain size.

ECoG is considered the gold standard for transducing electrical activity from the cerebral cortex. Brain activity is generally observed from 1 to $100 \mathrm{~Hz}$ and can be categorized as delta, theta, alpha, beta, and gamma waves based on the frequency of the wave. Beta-gamma waves, 13$100 \mathrm{~Hz}$, are associated with neurological cognitive activity. Activity seen during less conscious states constitutes other brain wave activity, with alpha ranging from 8 to $13 \mathrm{~Hz}$, theta from 4 to $7 \mathrm{~Hz}$, and delta below $4 \mathrm{~Hz}$. Alpha waves are generally associated with relaxed and calm states, beta with active conscious thought, and theta with sleep, deep relaxation and day dreaming, while delta is observed only during deep unconscious sleep [3].

Power spectral density (PSD) analysis is a method for evaluating the distribution of power in a signal over a range of frequencies. PSD is commonly used to determine levels of brain activity in ECoGs and electroencephalograph (EEG) recordings to quantitatively assess the power of each frequency observed in both conscious and unconscious states. Due to the identified spatio-temporal dynamics of ECoG recordings, the PSD of the signal often exhibits a linearly decreasing behavior over log-based coordinates with respect to frequency and amplitude of PSD or spectral power. This is called in the literature "power law" or scale-free behavior " $1 / f^{\alpha}$," where cognitive processing states vary by " $\alpha$ " [4]. The power law relation is measured over the whole range of frequencies from 1 to $100 \mathrm{~Hz}$, incorporating brain frequency distributions over delta, theta, alpha, beta, and gamma ranges. Power law behavior is attributed to the brain structural connectivity and dynamical properties.

For different human neurological brain states such as rest/sleep, awake, cognitive task engagement, and seizures, the PSD conform to the power law relation, $1 / f^{-\alpha}$, where $\alpha=-3$ when the patient is at rest, $-3<\alpha<-2$ during the awake state, $\alpha=-2$ during cognitive tasks and intentional behaviors [5-7]. The case, $\alpha=-2$ is mathematically referred to as brown noise, $1 / f^{-\alpha}$, whereas, $\alpha=-3$ is called black noise. The case, $a<-4$ occurs during the seizure state. The PSD during seizure likewise often differs sharply from the $1 / f$ form of the PSD in the rest and sleep states with high power in the theta ranges $<-4$ corresponding to the $3 / \mathrm{s}$ wave that dominated the EEG. Dominant power in the higher frequency ranges (20-80 $\mathrm{Hz}$ ) correspond to beta-gamma activity.

In animal cognition, gamma activity of sensory cortices has been shown to serve as a carrier wave for cognitive

PSD Analysis of ECoG Recordings during $\mathrm{CH}$ in NS information. Neural activity extends well beyond pairs of local areas of the cortex; neural signaling must reach all areas of the brain via higher narrow-band (beta-gamma) transmission [8-11].

Investigations involving changes of resting-state neuronal oscillations in the theta, alpha, and beta bands have found dominant power within these bands and change over the lifespan of an individual. Brain maturation measurement in the study by Boersma et al. utilized additional measurement techniques to complement power distribution per frequency changes, where principle component analysis have shown that power fluctuations across the scalp finally centered on a local neural neighborhood location [12, 13]. Power-law analysis of brain signals has gained interest, because it may reflect emergent population dynamics in critical systems [14-19]. Dynamic systems in a critical state are characterized by non-linearly interacting elements that organize into meta-stable spatial and temporal patterns. The idea of self-organized criticality provides the framework of non-linear dynamic systems converging into highly organized, correlated, neural activity patterns [20-22].

This study focuses on the neurological states captured via $\mathrm{ECoG}$ recordings from the parietal area of the cortex. Numerous experiments in non-human primate models reveal activation of the parietal cortex in a variety of cognitive functions, such as numerosity [23], categorization [24], and decision-making [25]. EEG and MEG studies have yielded consistent evidence on the time course of parietal mnemonic activation [26]. The conclusion that emerges from these studies is that the parietal cortex is activated in a wide range of tasks, and individual parietal neurons exhibit neural correlates of complex cognitive functions.

The goal of this study is to determine whether $\mathrm{CH}$ intervention can preserve neurological activity during seizure states using power law analysis [27-30], and specifically, whether higher frequency components, that is, beta-gamma activity $(13-100 \mathrm{~Hz})$ associated with cognition are preserved [31-34]. Higher power in the $3-4 \mathrm{~Hz}$ signal has been associated with seizure occurrence [35], therefore we would expect a higher peak at 3-12 Hz frequency range across the frequency spectrum, yielding a slope value where $\alpha<-4$. Variations in low frequency $3-12 \mathrm{~Hz}$ and high frequency $13-100 \mathrm{~Hz}$, will be measured in this study.

\section{Methods}

\section{Preparation}

Twelve piglets were utilized in this study, which was governed under IACUC guidelines (IACUC approval date: 07-09-2014; 
Animal Welfare Assurance Number A3325-01). Piglets were sedated and anesthetized using ketamine $(2 \mathrm{~mL}$ of $100 \mathrm{mg} / \mathrm{mL})$ and xylazine $(0.2 \mathrm{~mL}$ of $10 \mathrm{mg} / \mathrm{mL})$ via sterile syringes. Intravenous catheters were inserted into the femoral artery and vein for delivery of chemical agents. A tracheal tube was installed to regulate respiration via mechanical ventilation. Blood gas levels were adjusted to a normal range thereafter.

The head was secured in a stereotaxic unit and 2 stainless steel screws were installed in the parietal bone to serve as electrodes. Screws were placed $2.5 \mathrm{~cm}$ apart, each penetrating the skull to contact the underlying dura mater.

A single, differential ECoG signal was recorded from the 2 electrodes. Alligator clamps were used to attach both leads, and a reference electrode placed in the mouth of the animal. As this signal was on the scale of $\mu \mathrm{V}$, an amplifier (World Precision Instruments differential amplifier) with a gain set to 1,000 was used to boost the signal to a detectable level. Conversion from analog to digital form was performed at a sampling rate of $250 \mathrm{~Hz}$ using National Instruments (NI) cDAQ system (model 9172), which relayed output to the NI LabVIEW 2011 program for data processing and storage.

\section{Experimental Data Acquisition}

The baseline ECoG data was recorded for 30 min prior to seizure induction. Systemic parameters consisting of temperature, heart rate, and blood pressure were also documented at $5 \mathrm{~min}$ intervals during this time period. Temperature was assessed via rectal thermometer. Heart rate and blood pressure were displayed on an ECG monitor. Animals in the $\mathrm{CH}$ (cooled) group were subjected to localized hypothermia using ice packs placed on the head of the animal. This was done $10 \mathrm{~min}$ into baseline recordings to depress the temperature prior to seizure onset. Piglets were kept warm using a commercial heating pad throughout the experiment. Core body temperature was maintained at $37-38^{\circ} \mathrm{C}$ and $34-35^{\circ} \mathrm{C}$ in the control and $\mathrm{CH}$ groups, respectively.

Intravenous administration of paralytic pancuronium bromide $(0.5 \mathrm{~mL}, 1 \mathrm{mg} / \mathrm{mL})$ was used to block muscular activity during seizure events, as this would compromise data acquisition. Bicuculline (3 mg/kg i.v.), a GABA antagonist, was administered to induce seizures at the end of baseline recordings. Two hours of ECoG and systemic data were then recorded. Following ECoG recording, potassium chloride was administered to euthanize the piglet.

\section{Data Analysis}

ECoG data were assessed using MATLAB R2011a. Data was notch filtered at $60 \mathrm{~Hz}$ to remove the ambient noise in the signal, and linear regression was then applied across 2 groups at 2 different frequency ranges, low $(3-12 \mathrm{~Hz})$ and high $(13-100 \mathrm{~Hz})$. The last $72 \mathrm{~min}$ of the ECOG signal was analyzed, and the PSD was processed in non-overlapping 3-min windows. The slope of the power spectrum within each 3 -min window with respect to the frequency range in logarithm scale was calculated as shown in equation 1.

$$
\text { Slope }(\alpha)=\frac{d B}{\log _{10}(\mathrm{~Hz})}
$$

This slope assessment condenses information about the frequency distribution into a single value, such as seizure activity and enables the construction of a simple 2-dimensional graph to describe PSD across all time segments of a signal.
Average slopes and SDs from cooled and non-cooled groups were compared. Statistical analysis with $p<0.05$ (considered significant) was performed for each assessed frequency value with unpaired, 2-tailed Student $t$ test, and compared between groups. Asterisks indicate significance, ${ }^{* * *} p \leq 0.01$. Variance was calculated for low/high frequency ranges of their respective slopes.

\section{Results}

\section{Analysis of PSD Slope Values}

Slope values for each individual animal in both $\mathrm{CH}$ (cooled) animals $(n=6)$ and control animals $(n=6)$ were calculated by applying linear regression across 3-12 and 13-100 Hz. Figure 1 shows slopes for representative animals in the $\mathrm{CH}$ and control groups. The slope averages and SDs are shown in Figure 2. Slopes were significantly different between $\mathrm{CH}$ and non- $\mathrm{CH}$ cohorts in the higher frequencies $(p<0.01)$ after 50 min of seizure. Beta-gamma activity seems to remain fixed around $\sim 3.5 \mathrm{~dB} / \log _{10} \mathrm{~Hz}$ in the cooled group (Fig. 2b) over time.

\section{Percentage of Change of Low/High Frequency Ranges between Cohorts}

Our goal is to find the highest degree of change within the frequency domain. Thus, we calculate the variance of the cohorts low and high frequency ranges by taking the slope of power spectrum from 3 to 12 and 13-100 Hz and derive the variance of the average trials. Results show that the variance is lower in the lower frequency range (3$12 \mathrm{~Hz}$ ) among the control and cooled cohort towards the end of the time series (Fig. 3a) compared to the higher frequency range $(13-100 \mathrm{~Hz})$, where the cooled cohort has a lower variance compared to the control group, which has a much higher variance (Fig. 3b).

\section{Assessment of 3 Min Interval Segments}

Our results suggest that the overall power over the frequency spectrum decreases as seizure continues, whereas the power in the cooled group over the higher frequency ranges appears to remain fixed after a period of time, preserving the beta-gamma range of brain activity.

\section{Discussion}

\section{Analysis of PSD Slope Values}

Directional shifts between high and low frequencies are associated with changes in cerebral blood flow and metabolism. Detection of state transitions in EEGs is important in brain studies as it relates to mechanisms of loss 

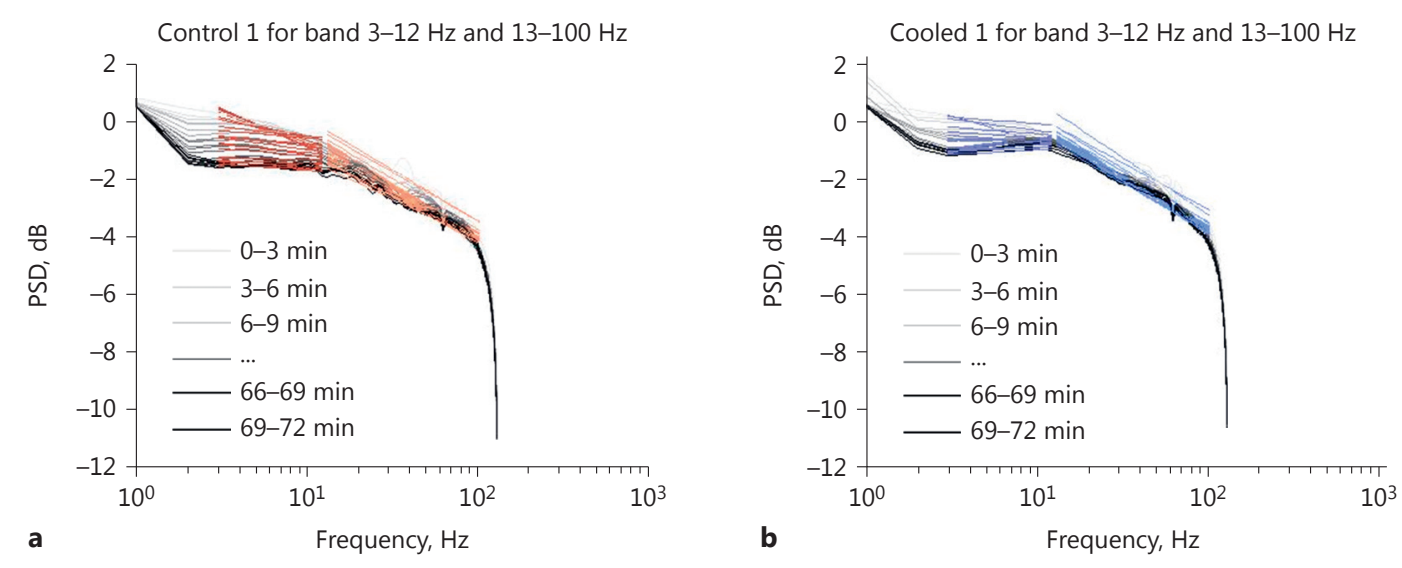

Fig. 1. PSD changes across time for the control group (a) and the cooled group (b). Linear regression is applied to 3-12 and 13-100 Hz ranges to quantify changes across the frequency spectrum. Both groups (a, b) exhibit an overall decrease of power across the frequency spectrum.

Fig. 2. Time-dependent changes in PSD slope (mean and SD) between cooled (blue) and control (red) groups, where ${ }^{* * *} p<$ 0.01). a 3-12 Hz PSD slope values; b 13$100 \mathrm{~Hz}$ PSD slope values.

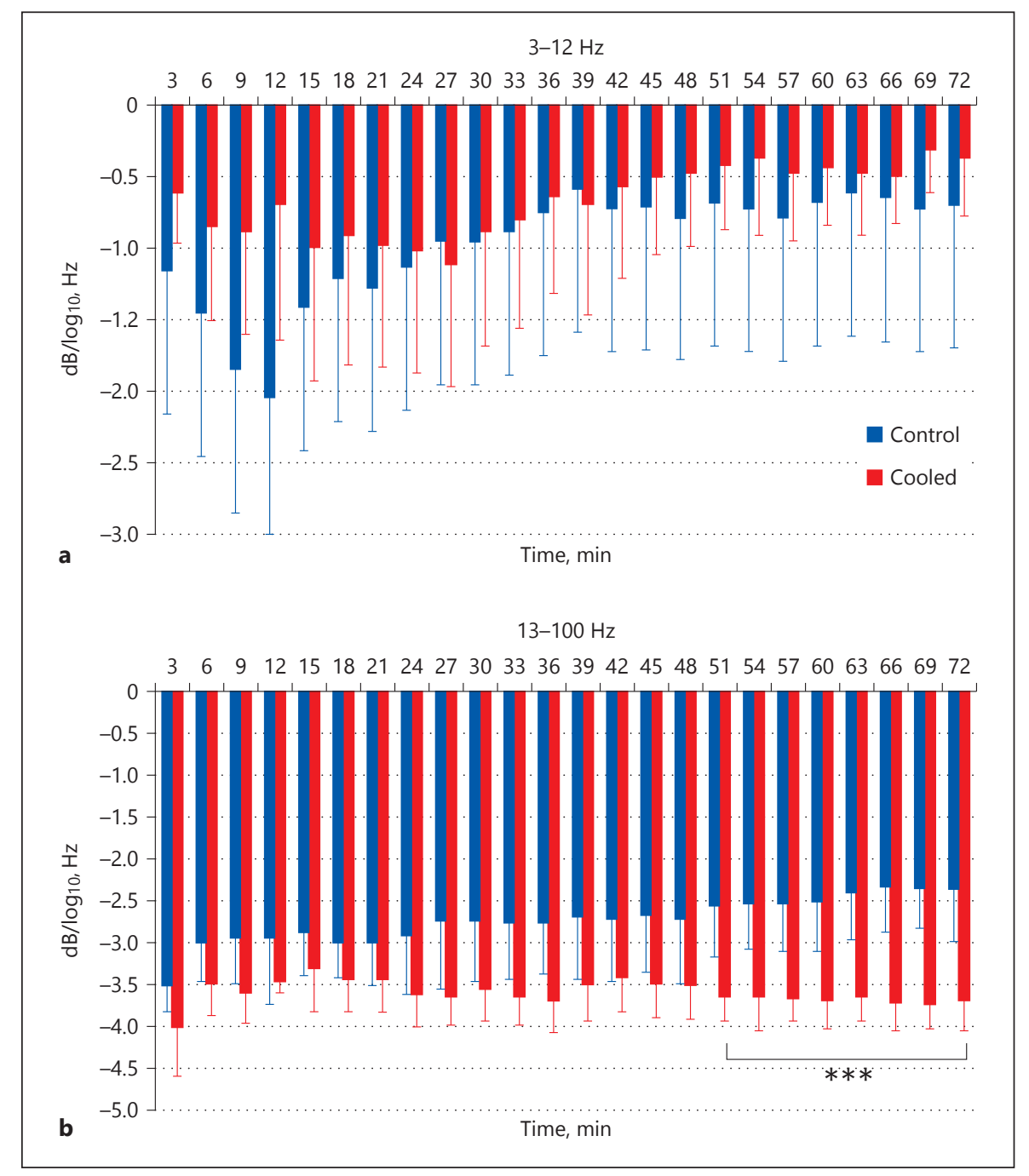




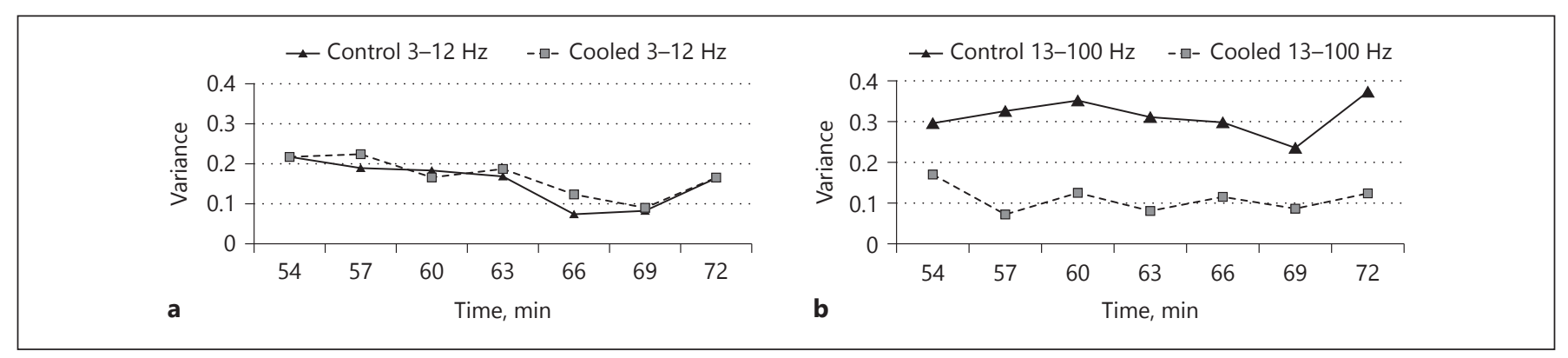

Fig. 3. Control and cooled group comparisons in (a) 3-12 Hz where both variances decrease towards the end of the time series. Variances of the cooled group remain fixed (b) where the control group maintains high variability at $13-100 \mathrm{~Hz}$ ranges.

of stability in neural populations due to brain disease or trauma. Changes in cerebral autoregulation has been attributed to the increase in blood pressure associated with seizure activity [36], Functional brain imaging experiments have confirmed the relationship between blood flow and a sudden change of function in a portion of the brain connected to a distant, but pathological brain area, known as cerebellum diaschisis [37]. Therefore, there is a reallocation of power from a localized neural population site to other neural populations across the cortex. Specifically, increases or reduction of power within a frequency range, in this case from long-term seizure activity may be due to a reallocation of blood flow which affects the brain functionality.

Our analysis is used to better understand the underpinnings of neural homeostasis, that is, background neural firing related to normal electrical brain behavior. This analysis has been utilized to understand the abnormal "unbalanced" neural behavior as it relates to runaway neural activity that can dominate the entire cortex of the brain (such as seizures).

A typical PSD display features a diminishing sinusoidal waveform pattern, where dominant frequencies reside in the beta-gamma brain frequency range $(20-80 \mathrm{~Hz})$, which represents cognitive activity. Linear regression is performed on PSD values, where the slope is taken across the power spectrum which may show higher or lower power in this area due to a redistribution of power from other dominant areas across the power spectrum. Figure 2 contains data from direct analysis of slope values, which describe the overall trends of power distributions seen across the frequency spectrum. The slope of the control group tends to lower power/frequency over time, whereas the cooled group appears to remain stable after $50 \mathrm{~min}$, with 3.6.

The control group appears to have a reduced power due to ongoing seizure, thereby reducing the overall neu- ral activity. The cooled group maintains a relatively high power over frequency ranges, but this activity may be dominated by ongoing seizure behavior. Most importantly, neural activity remains stable over time, preserving high neural activity, also demonstrated in Figure $2 b$. Figure $2 \mathrm{~b}$ illustrates stable power/frequency averaged slope values in the cooled group compared to the control group, with relatively stable SD. High SD seen in Figure 2a and $b$ may be mitigated through a larger number of subjects in future studies.

\section{Percentage of Change of Low/High Frequency \\ Ranges between Cohorts}

To determine where $\mathrm{CH}$ had the most noticeable effects on the cooled group in terms of brain activity, the SD was divided by the average of all the recordings per cohort, as seen in Figure 3. Trends of cooled and control groups plotted together demonstrate little difference between high $13-100 \mathrm{~Hz}$ frequency ranges. While some variability is seen in the low frequency of the control group, a much higher degree of variability is seen in the low frequency $(3-12 \mathrm{~Hz})$ of the cooled group. This variability placed together with the plotted average slopes in Figure 2 demonstrates that while the seizure state dominates neural behavior, the greatest amount of change seems to be on the overall decrease of power across the frequency spectrum, as seen in Figure $1 \mathrm{a}$ and $\mathrm{b}$.

\section{Assessment of 3 Min Interval Segments}

PSD slopes were more closely examined to confirm observations made in Figures 1 and 3. Tables 1 and 2 contain data analysis from the last $72 \mathrm{~min}$ of seizure recordings. Table 1 contains $t$ score probabilities and SD and variance for $3-13 \mathrm{~Hz}$ low frequency ranges, which demonstrate no difference in slopes at any given time; 
Table 1. Means and SDs for PSD slope values (3-12 Hz range)

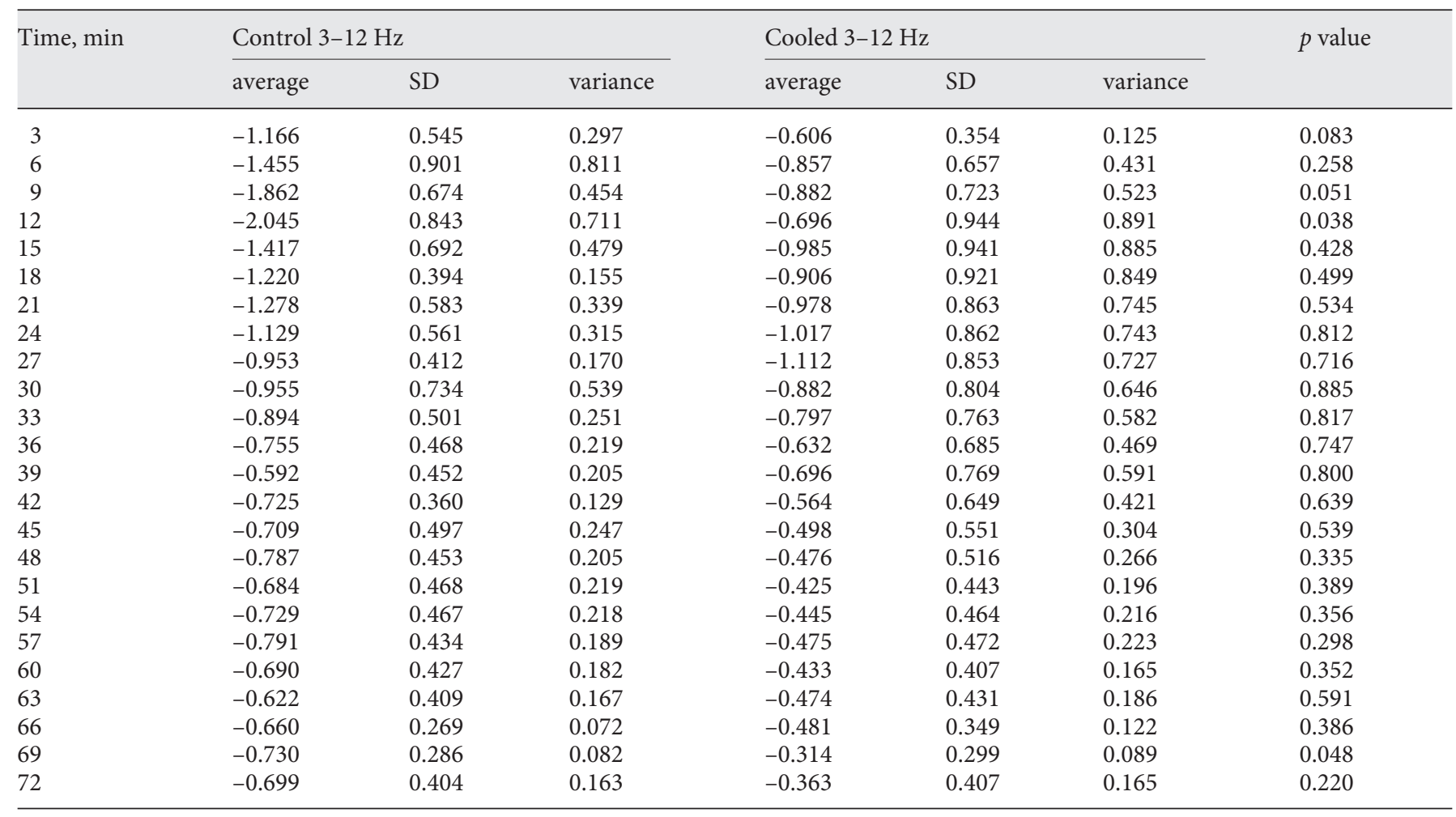

Table 2. Means and SDs for PSD slope values (3-100 Hz range)

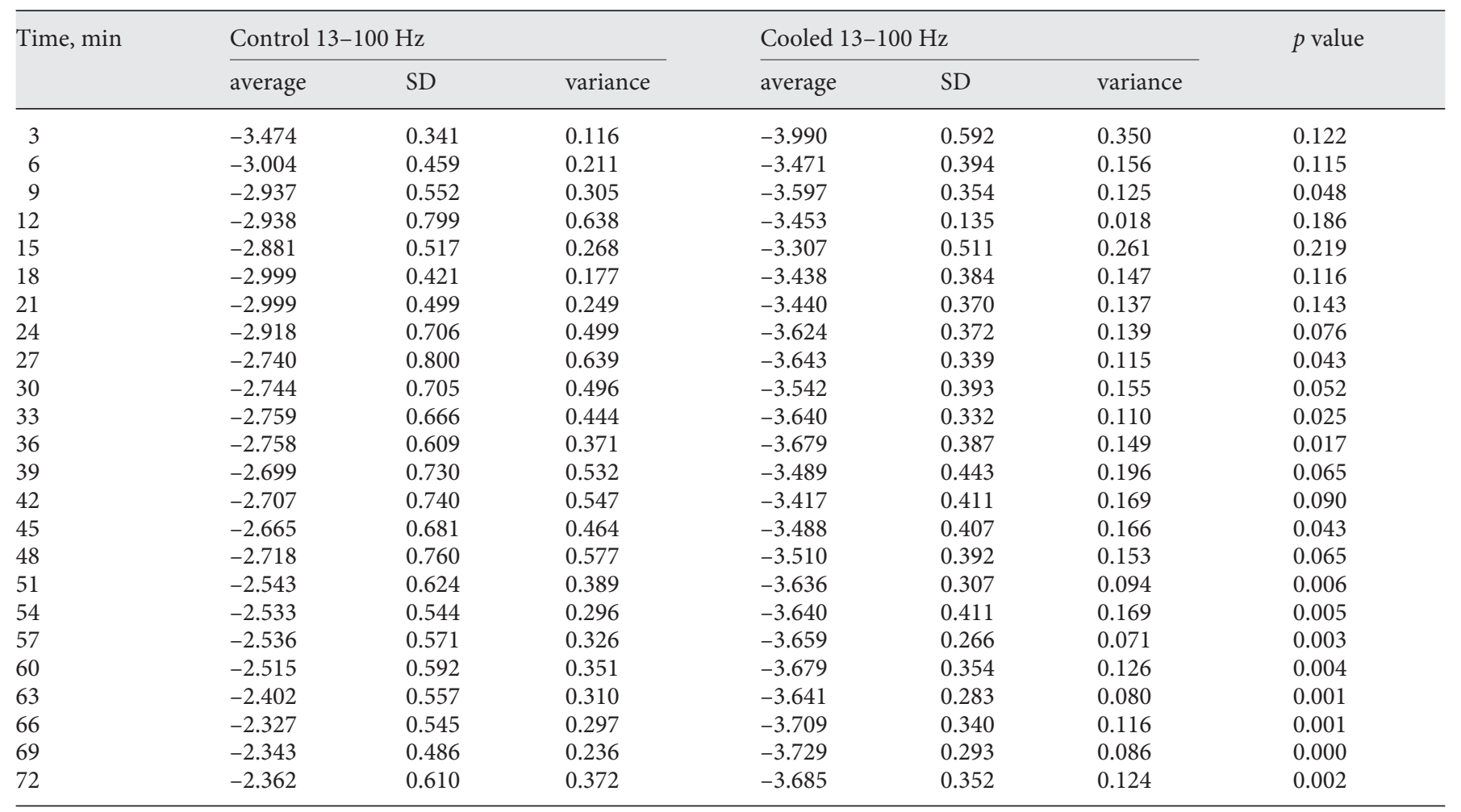


therefore, there is no statistical difference between the cohorts.

Table 2 presents high frequency analysis, featuring latter intervals in time where $p$ values approach a high level of significance. For high frequency ranges between control and cooled cohorts, from 24 to $51 \mathrm{~min}$, there is a relatively significant difference between the cohorts $(0.05<p<0.1)$, and from 51 to $72 \mathrm{~min}$, there are significant differences between the cohorts $(p \leq 0.01)$. We also see relatively small variances during the same time period, demonstrating that there is very little change in neural activity during head cooling, while high variances are seen in the control group. Both $p$ values and variances in this time period are in bold. The second hour's data focuses on specific frequency boundaries, whereas, high frequency ranges exhibit a clear delineation between the cohorts.

Tables 1 and 2 juxtapose the variances with $p$ values to show that the highest separation between the groups and the variability of the power distribution across the frequency spectrum occurs in the higher frequencies of the control group, while lower frequencies change very little over time. Altogether, these tables demonstrate that the overall behavior of neural activity between the groups; the control groups exhibits the sharpest overall decline and variability of neural activity during seizure induction, while the cooled group's neural activity remains stable towards the last $20 \mathrm{~min}$ of the experiment.

To answer the following questions, "can $\mathrm{CH}$ intervention preserve neurological activity during seizure states," we have seen from Figure 2 that high slope values are maintained as seizure activity persists, while Figure 3 demonstrates little changes in the high frequency range during cooling. While there appears to be an overall decrease of neural activity, the rate of change occurs for the most part in the high frequency range of the control group; therefore, neural activity is preserved during induced seizure states. Additionally, since the higher frequency brain activity is related to beta-gamma neural frequency range, we also suggest that $\mathrm{CH}$ therapy helps to preserve the neural activity related to cognitive behavior. Therefore, head cooling appears to preserve the brain functionality from cerebral vascular injury caused by seizures.

\section{Conclusion}

Statistical investigations on ECoG data has shown that $\mathrm{CH}$ helps to preserve the cognitive functionality observed in higher frequency range during seizure. $\mathrm{CH}$ intervention controls the degree of neural activity fluctuations over time, leading to better control of brain activity from the influence of induced seizures, while non- $\mathrm{CH}$ intervention has shown a decrease of activity over time. Within the control group, the signals presented high variability in the higher frequencies and low variability in the low frequencies, where neural activity drops overall over time. Conversely, the $\mathrm{CH}$ group exhibits high variability in lower frequencies, and low variability in high frequencies, demonstrating that neural activity decreases at a much slower pace, extending the duration of neural activation, and specifically preserving the cognitive functionality. Additional experiments will be needed to provide further evidence that $\mathrm{CH}$ may serve as a preventative measure against the effects of NS.

\section{Acknowledgements}

The authors wish to thank Alex Fedinec for performing surgical procedures involved in this experiment.

\section{Author Contributions}

M.H.M. developed the algorithm, literature review, statistical analysis and developed the manuscript; H.P. developed the experiment; E.J. and Y.L. rigorously tested the algorithm; A.J.C. and H.P. provided additional analysis and editing of the manuscript.

\section{Disclosure Statement}

The author declares no conflict of interest. The manuscript is complied with International Committee of Medical Journal Editor's guidelines.

\section{Disclaimer}

The views expressed in the submitted article are his or her own and not an official position of the institution.

References

1 Sheth R: Neonatal Seizures. Mayo College of Medicine, Medscape References. http:// emedicine.medscape.com/article/1177069overview (accessed June 10, 2014).

2 Gunn AJ, Gunn TR, Gunning MI, Williams CE, Gluckman PD: Neuroprotection with prolonged head cooling started before postischemic seizures in fetal sheep. Pediatrics 1998; 102:1098-1106.

3 Brain and Health: The Basics of Brain Waves. http://www.brainandhealth.com/BrainWaves.html (accessed January 5, 2012).
18

Ann Neurosci 2017;24:12-19

DOI: $10.1159 / 000464418$ 
4 Freeman WJ: Origin, structure, and role of background EEG activity. Part 4. Neural frame simulation. Clin Neurophysiol 2006; 117:572-589.

5 Freeman WJ, Holmes MD, West GA, Vanhatalo S: Dynamics of human neocortex that optimizes its stability and flexibility. J Intellignt Sys 2006;21:881-901.

6 Kelso S, Tognoli E: Toward a complementary neuroscience: metastable coordination dynamics of the brain. Neurodyn Cogn Conscious 2007;Part 1:39-59.

7 Lachaux JP, George N, Tallon-Baudry C, Martinerie J, Hugueville L, Minotti L: The many faces of the gamma band response to complex visual stimuli. NeuroImage 2005;25: 491-501.

8 Haig AR, Gordon E, Wright JJ, Meares RA, Bahramali H: Synchronous cortical gammaband activity in task-relevant cognition. Neuroreport 2000;11:669-675.

9 Barrie JM, Freeman WJ, Lenhart M: Modulation by discriminative training of spatial patterns of gamma EEG amplitude and phase in neocortex of rabbits. J Neurophysiol 1996;76: 520-539.

10 Freeman WJ, Barrie JM: Analysis of spatial patterns of phase in neocortical gamma EEGs in rabbit. J Neurophysiol 2000;84:1266-1278.

11 Takeshita S, Sato Y, Bahar S: Transitions between multistable states as a model of epileptic seizure dynamics. Phys Rev E Stat Nonlin Soft Matter Phys 2007;75(5 pt 1):051925.

12 Lehmann D, Strik WK, Henggeler B, Koenig T, Koukkou M: Brain electric microstates and momentary conscious mind states as building blocks of spontaneous thinking. I. Visual imagery and abstract thoughts. Int J Psychophysiol 1998;29:1-11.

13 Boersma M, Smit DJ, de Bie HM, Van Baal GC, Boomsma DI, de Geus EJ, Delemarre-van de Waal HA, Stam CJ: Network analysis of resting state EEG in the developing young brain: structure comes with maturation. Hum Brain Mapp 2011;32:413-425.

14 Bak P, Tang C, Wiesenfeld K: Self-organized criticality: an explanation of the $1 / \mathrm{f}$ noise. Phys Rev Lett 1987;59:381-384.

15 Bak P, Paczuski M: Complexity, contingency, and criticality. Proc Natl Acad Sci U S A 1995; 92:6689-6696.
16 Chialvo DR: The brain near the edge; in Marro J, Garrido PL, Torres JJ (eds): Cooperative Behavior in Neural Systems: Ninth Granada Lectures. Melville, American Institute of Physics, 2007.

17 Levina A, Herrmann JM, Geisel T: Dynamical synapses causing self-organized criticality in neural networks. Nat Physics 2007;3:857860.

18 Poil SS, van Ooyen A, Linkenkaer-Hansen K: Avalanche dynamics of human brain oscillations: relation to critical branching processes and temporal correlations. Human Brain Mapp 2008;29:770-777.

19 Kello CT, Brown GD, Ferrer-I-Cancho R, Holden JG, Linkenkaer-Hansen K, Rhodes T, Van Orden GC: Scaling laws in cognitive sciences. Trends Cogn Sci 2010;14:223-232.

20 Linkenkaer-Hansen K, Nikouline VV, Palva JM, Ilmoniemi RJ: Long-range temporal correlations and scaling behavior in human brain oscillations. J Neurosci 2001;21:1370-1377.

21 Freeman WJ: Origin, structure, and role of background EEG activity. Part 2. Analytic phase. Clin Neurophysiol 2004;115:20892107.

22 Plenz D, Thiagarajan TC: The organizing principles of neuronal avalanches: cell assemblies in the cortex? Trends Neurosci 2007;30: 101-110.

23 Roitman JD, Brannon EM, Platt ML: Representation of numerosity in posterior parietal cortex. Front Integr Neurosci 2012;6:25

24 Fitzgerald JK, Swaminathan SK, Freedman DJ: Visual categorization and the parietal cortex. Front Integr Neurosci 2012;6:18.

25 Huk AC, Meister ML: Neural correlates and neural computations in posterior parietal cortex during perceptual decision-making. Front Integr Neurosci 2012;6:86.

26 Levy DA: Towards an understanding of parietal mnemonic processes: some conceptual guideposts. Front Integr Neurosci 2012;6:41

27 Martin WR, Raichle ME: Cerebellar blood flow and metabolism in cerebral hemisphere infarction. Ann Neurol 1983;14:168-176.
28 Myers MH, Kozma R: Seizure Prediction through Dynamic Synchronization Measures of Neural Populations. Proc Int J Conf Neur Netw, IJCNN09, June 14-19, 2009 in Atlanta, Georgia, pp 2195-2201, IEEE Press.

29 Myers MH, Kozma R: Modeling Normal/ Epileptic Brain Dynamics with Potential Application in Titration Therapy. Proc, IEEE/ INNS Joint Conference on Neural Networks, IJCNN11, July 31-August 5, 2011, San Jose, California.

30 Chang HJ, Freeman WJ, and Burke BC: Optimization of olfactory model in software to give $1 /$ f power spectra reveals numerical instabilities in solutions governed by aperiodic (chaotic) attractors. Neural Netw 1998;11: 449-466.

31 Freeman WJ: Petit mal seizure spikes in olfactory bulb and cortex caused by runaway inhibition after exhaustion of excitation. Brain Res 1986;396:259-284.

32 Bressler SL, Kelso JA: Cortical coordination dynamics and cognition. Trends Cogn Sci 2001;5:26-36.

33 Bertrand O, Tallon-Baudry C: Oscillatory gamma activity in humans: a possible role for object representation. Int J Psychophysiol 2000;38:211-223.

34 Dumenko VN: The functional significance of high-frequency components of brain electrical activity; in Miller R, Ivanitzky AM, Balaban PM (eds): Complex Brain Functions. Complex Brain Functions: Conceptual Advances in Russian Neuroscience. Amsterdam, Taylor \& Francis, Inc., 2000, pp 129-150.

35 Myers MH, Kozma R: Modeling Dynamics of the Human Limbic System. The 3rd International Conference on Cognitive Neurodynamics, ICCN11, June 9-11, 2011, Hokkaido, Japan.

36 Smit DJ, de Geus EJ, van de Nieuwenhuijzen $\mathrm{ME}$, van Beijsterveldt CE, van Baal GC, Mansvelder HD, Boomsma DI, LinkenkaerHansen K: Scale-free modulation of restingstate neuronal oscillations reflects prolonged brain maturation in humans. J Neurosci 2011; 31:13128-13136.

37 Raichle ME: Behind the scenes of functional brain imaging: a historical and physiological perspective. Proc Natl Acad Sci U S A 1998; 95:765-772. 